\title{
Incidence of occupational latent tuberculosis infection in South African healthcare workers
}

\author{
Shahieda Adams ${ }^{1,2}$, Rodney Ehrlich ${ }^{2}$, Roslynn Baatjies ${ }^{3}$, Richard N. van Zyl-Smit ${ }^{1}$, \\ Qonita Said-Hartley ${ }^{4}$, Rodney Dawson ${ }^{5}$ and Keertan Dheda ${ }^{1}$
}

Affiliations: ${ }^{1}$ Lung Infection and Immunity Unit, Dept of Medicine, University of Cape Town, Cape Town, South Africa. ${ }^{2}$ Centre for Environmental and Occupational Health Research, School of Public Health and Family Medicine, University of Cape Town, Cape Town, South Africa. ${ }^{3}$ Dept of Environmental and Occupational Studies, Faculty of Applied Sciences, Cape Peninsula University of Technology, Cape Town, South Africa. ${ }^{4}$ Radiology Dept, New Somerset Hospital, Cape Town, South Africa. ${ }^{5}$ Centre for TB Research Innovation, University of Cape Town Lung Institute, Cape Town, South Africa.

Correspondence: Keertan Dheda, Lung infection and Immunity Unit, Dept of Medicine, University of Cape Town H46.41, Old Main Building, Groote Schuur Hospital, Observatory, 7925, South Africa.

E-mail: keertan.dhedaduct.ac.za

ABSTRACT The test-specific incidence of latent tuberculosis infection (LTBI) in healthcare workers from sub-Saharan Africa is unknown.

505 healthcare workers from South Africa were screened at baseline, and after 12 months, with a questionnaire, the tuberculin skin test (TST), and two T-cell assays (T-SPOT.TB and QuantiFERON-TB Gold-In-Tube). Test-specific conversion rates were calculated.

The prevalence of presumed LTBI at baseline was 84, 69 and $62 \%$ using the TST, QuantiFERON-TB Gold-In-Tube and T-SPOT.TB, respectively. The annual test-specific conversion rate, depending on the cut-off point used, was as follows: TST 38\%; QuantiFERON-TB Gold-In-Tube 13-22\%; and T-SPOT.TB 18-22\%. Annual reversion rates were 4,7 and $16 \%$, respectively. The annual TST conversion rate was significantly higher than that derived from published local community-based data (IRR 3.53, 95\% CI 1.816.88). Factors associated with conversion (any test) included healthcare sector of employment, counselling of tuberculosis patients, and a baseline positive TST (for T-SPOT.TB).

The annual rate of tuberculosis infection in South African healthcare workers was very high, irrespective of the testing method used, and may be explained by occupational exposure, as the rate was considerably higher than non-healthcare workers from the same community. Collectively, these data support the need for implementation of tuberculosis-specific infection control measures in Africa.

@ERSpublications

South African healthcare workers have a high incidence of latent tuberculosis infection due to occupational exposure http://ow.ly/HDXhH

This article has supplementary material available from erj.ersjournals.com

Received: July 292014 | Accepted after revision: Dec 092014 | First published online: Feb 192015

Support statement: This study was funded by grants from the South African Medical Research Council, National Institute of Health (Fogarty International Clinical Research Fellowship (R24 TW007988-03) and SATBAT (IU2RTW007370-01A1)) and European Developing Clinical Trials Partnership (EDCTP TB NEAT: IP_09_32040_009). Funding information for this article has been deposited with FundRef. Kits and tubes for QuantiFERON ${ }^{-}$-TB Gold-In-Tube testing were sponsored by Cellestis, whilst T-SPOT.TB ${ }^{\oplus}$ Test kits were sponsored by Oxford Immunotech. Neither Cellestis, nor Oxford Immunotech had any role in the design and conduct of the study; collection, analysis or interpretation of data; and preparation, review or approval of the manuscript.

Conflict of interest: Disclosures can be found alongside the online version of this article at erj.ersjournals.com

Copyright (CERS 2015 


\section{Introduction}

Healthcare workers are considered a high-risk group for contracting occupational tuberculosis (TB) [1-4]. Based on limited data the median annual incidence of active TB among healthcare workers in TB endemic countries is approximately 1180/100000 (interquartile range (IQR) 91-3222) [2]. An increasing incidence of active TB (both drug sensitive and resistant) in healthcare workers (and TB field researchers and community workers) has been demonstrated in tandem with a growing HIV epidemic in South Africa [5-9].

Retention of skilled healthcare workers in the public health system in these countries is crucial to the implementation of programmes that address the large TB and HIV burden [10]. This consideration has resulted in advocacy for more effective prevention, diagnosis, and management of TB and HIV in healthcare workers [11]. Implementation of infection control measures, allocation of resources, and rational planning of intervention strategies require accurate estimates of the scale of the problem. Moreover, the success of preventative strategies, such as targeted prophylaxis, needs to be measured through changes in the incidence of latent tuberculosis infection (LTBI) and disease progression. However, although the median annual risk of new LTBI in healthcare workers in high incidence countries is estimated to be $7.2 \%$ (IQR 4.1-14.3\%), there are hardly any data from Africa to inform these estimates [12].

Traditionally, screening for LTBI was performed using the tuberculin skin test (TST). More recently, interferon- $\gamma$ release assays (IGRAs) have been used for the diagnosis of LTBI. There are two commercially available IGRA assays: the T-SPOT.TB assay (Oxford Immunotech, Abingdon, UK) and the QuantiFERON-TB Gold-In-Tube (QFT-GIT) assay (Cellestis Ltd, Carnegie, Australia). These assays have some advantages over the TST, but also several drawbacks, such as the need for laboratory expertise and infrastructure and their relatively high cost [13]. They have been incorporated into testing guidelines in countries such as the USA and UK, where serial LTBI testing of healthcare workers is routinely performed $[14,15]$. However, little research has focused on their potential value in healthcare workers from TB endemic countries. Moreover, annual rates of new TB infection in healthcare workers in Africa using different testing methods have hitherto not been determined.

To address these gaps in our knowledge we undertook a prospective study among healthcare workers in South Africa with the specific objectives of: 1) evaluating the prevalence of presumed LTBI using three different testing modalities (TST, T-SPOT.TB and QFT-GIT); 2) determining the incidence of LTBI as measured by test-specific conversion rates; and 3) determining the occupational factors associated with TB infection.

\section{Methods}

Study setting and participants

A prospective cohort study was conducted over a 2-year period (May 2009 to July 2011) among healthcare workers employed at seven healthcare facilities in Cape Town, South Africa. The five primary healthcare facilities in the sample are community-based and offer diagnostic and treatment services for $\mathrm{TB}$, while the two secondary facilities are TB hospitals providing care to patients with complicated or drug-resistant TB. Staff were mainly employed by the provincial or local authority health departments, and the rest in the non-governmental sector. Recruitment took place sequentially over a year using a trained nurse to draw blood samples and administer the TST. Staff included both support (administrative, security and lay healthcare workers) and clinical staff (interns, researchers, trainees, nurses and doctors) over the age of 18 years. Those who were known to be pregnant or allergic to tuberculin were excluded from the study.

Participants completed an interviewer-administered questionnaire covering HIV status, and TB-associated and occupational and environmental risk factors. All underwent a digital chest radiograph, which was independently read by two physicians trained in the Chest Radiograph Review System (CRRS), a standardised epidemiological risk assessment tool for TB [16]. An expert chest radiologist resolved disagreement between the two readers. Those testing positive on symptom screen or chest radiograph were investigated further using sputum microscopy and culture in order to exclude active TB.

All participants were requested to have an HIV test, which was a rapid ELISA test performed in accordance with the manufacturer's instructions. A positive result was followed up with a confirmatory test using a rapid ELISA test from a different manufacturer, as per the study protocol. Participants were invited to report their HIV test result if previously tested. Refusal to disclose HIV status or undergo testing did not render participants ineligible for inclusion in the study.

The study was approved by the human research ethics committee of the Faculty of Health Sciences at the University of Cape Town, Cape Town, South Africa. All HIV infected individuals were referred for isoniazid prophylaxis and recommended for redeployment to a lower risk setting. Individuals newly diagnosed with active TB were referred for TB treatment. 


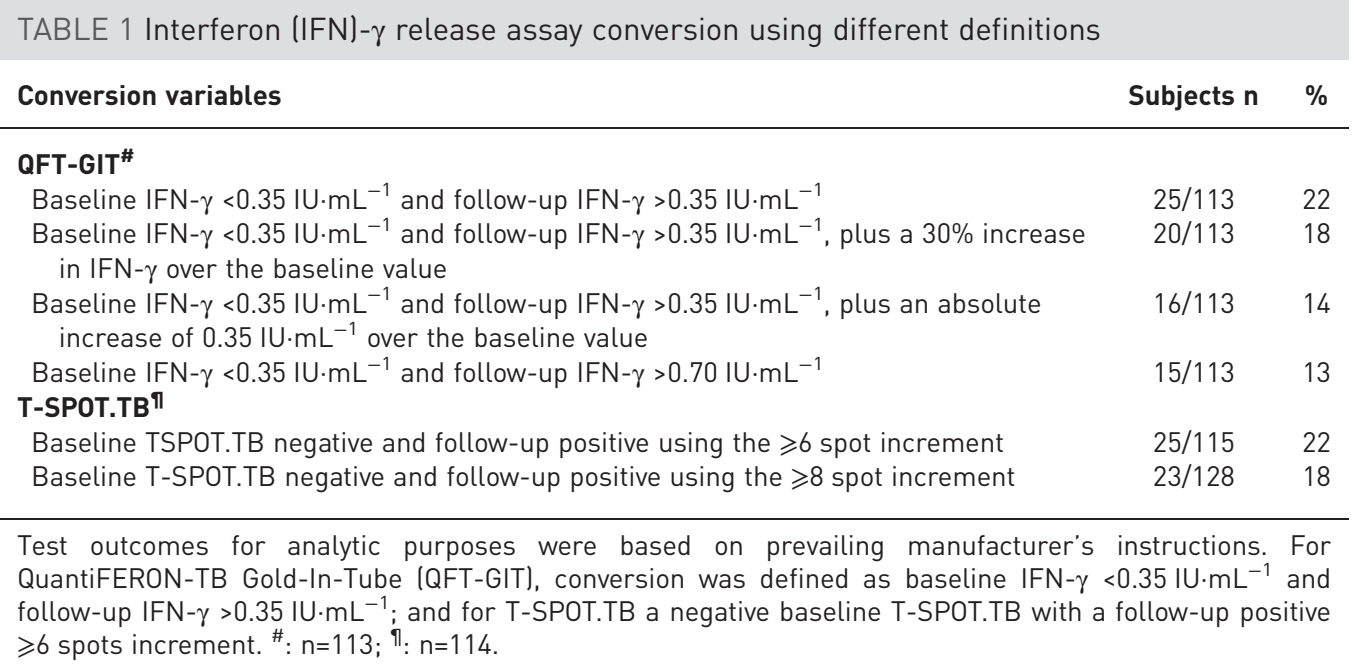

\section{TST testing and interpretation}

The one step TST protocol was employed using two tuberculin units $(0.1 \mathrm{~mL})$ of RT23 Purified Protein Derivative (PPD; Staten Serum Institute, Copenhagen, Denmark), injected intradermally on the volar aspect of the forearm. The induration was measured by a trained reader after $48-72 \mathrm{~h}$ using the ballpoint reader method. An induration of $\geqslant 10 \mathrm{~mm}$, or in the case of an HIV positive individual, $\geqslant 5 \mathrm{~mm}$, was considered a positive test at baseline.

\section{IGRA testing (QFT-GIT and T-SPOT.TB)}

Bloods for the IGRA assays (QFT-GIT and the T-SPOT.TB, performed according to the manufacturers' instructions) were drawn concurrently or within 3 days of administering the TST to eliminate any effect of potential boosting [17].

TST conversion was considered to occur if the baseline TST was classified as negative (induration of $<10 \mathrm{~mm}$ ), and follow-up positive TST as positive (induration $\geqslant 10 \mathrm{~mm}$ with an increase of at least $10 \mathrm{~mm}$ ) as per American Thoracic Society/Centers for Disease Control and Prevention and Infectious Diseases Society of America guidelines [18]. IGRA conversion was defined as a negative baseline test and a positive follow-up test. For QFT-GIT the required change was from a baseline interferon- $\gamma<0.35 \mathrm{IU} \cdot \mathrm{mL}^{-1}$ to a follow-up interferon- $\gamma>0.35 \mathrm{IU} \cdot \mathrm{mL}^{-1}$. For T-SPOT.TB a negative test at baseline changing to a positive test $(\geqslant 6$ spots increment in either panel $\mathrm{A}$ or $\mathrm{B})$ on follow-up testing was required. Various cut-off points for conversion were explored for both IGRAs (table 1). Reversion was defined as a baseline positive test with a negative test at follow-up.

\section{Statistical analysis}

Statistical analyses were performed using Stata version 11 (Stata Corp, College Station, TX, USA). The prevalence of TB-associated risk factors and test outcomes were calculated at baseline. Agreement between test outcomes was computed using the Kappa statistic. Annual rates of TST and IGRA conversion and predictors of conversion were evaluated. Risk factors for test positivity were evaluated using odds ratios with 95\% confidence intervals. Univariate logistic regression analysis was performed to evaluate the relationship between potential risk factors for TB infection and all three test outcomes. Separate multivariate regression models were then run for all variables of interest in relation to each test outcome, adjusting for covariates such as age and sex. A p-value of $<0.05$ was considered significant.

\section{Results}

\section{Demographic characteristics and participation}

In total 764 healthcare workers were eligible and 510 consented to be included in the study, representing a response rate of $67 \%$. The follow-up phase of the study commenced 1 year after the baseline with 339 healthcare workers followed up from the original cohort of 505, representing a follow-up rate of $67 \%$. The main reason for participants being lost to follow-up was leaving the facility (91 participants). Most had left for unknown reasons (68), three had resigned, three had retired and six were students who had completed their rotation, while another 11 had been transferred out (fig. 1). 
All participants completed the questionnaire. HIV results were available for 329 participants and chest radiographs for 288 participants. A substantial proportion (56\%) declined to have a repeat TST administered, so that only 142 participants had both a baseline and follow-up TST reading. Uptake of repeat IGRAs was very high with $99 \%$ of participants undergoing repeat testing. This resulted in 332 participants having valid paired results for QFT-GIT and 292 for T-SPOT.TB. The population predominantly consisted of females (71\%) with $58 \%$ over the age of 40 years (table 2). Duration of employment in a healthcare environment was $<10$ years for the majority of participants (53\%), while $30 \%$ reported a long duration of employment ( $>20$ years; table 2 ).

\section{Occupational and environmental characteristics}

Involvement in delivering healthcare services to TB patients ranged from $2 \%$ for bronchoscopy procedures to $49 \%$ for interviewing patients (table 3). High risk procedures, such as nursing of TB patients and collection of sputum samples, were performed by $30 \%$ and $22 \%$ of respondents, respectively. There was varying reported facility compliance with environmental control measures for TB infection control, with $64 \%$ of participants reporting that their facility had a specific infection control policy. Most displayed an awareness of ventilation measures being implemented at their facility (93\%), while reported compliance with the provision of personal protective equipment was high (89-95\%).

\section{Prevalence of TB associated risk factors and LTBI}

The prevalence of reported BCG vaccination (in childhood) was high at 92\% (table 4). Three participants who had previously been negative at baseline, had a positive HIV test at follow-up representing an HIV infection incidence of $1 \%$ (95\% CI 0.18-2.56). Two new cases of active TB were diagnosed at follow-up and one participant not previously diagnosed had died of TB in the interval, yielding a TB incidence of nine per 1000 (1\%, 95\% CI 0.2-2.6). LTBI prevalence was high at $84 \%$ for TST, and 69\% and 62\% for QFT-GIT and T-SPOT.TB, respectively.

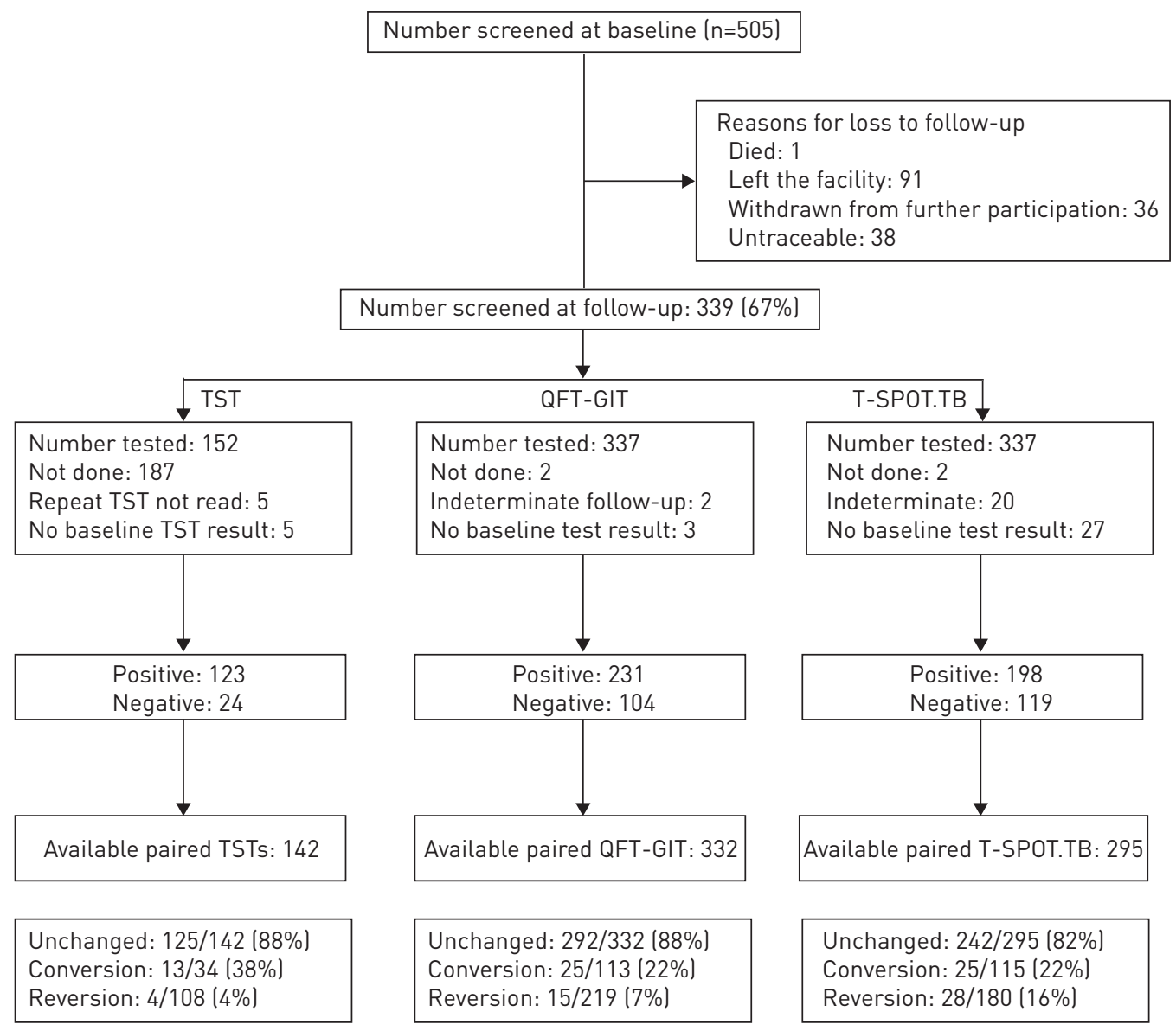

FIGURE 1 Healthcare worker cohort screened at follow-up for latent tuberculosis infection. TST: tuberculin skin test. QFT-GIT: QuantiFERON-TB Gold-In-Tube. 
TABLE 2 Characteristics of participants who underwent repeat testing for latent tuberculosis infection by either tuberculin skin test and/or interferon- $\gamma$ release assay

\begin{tabular}{|c|c|c|}
\hline Variable & Subjects $\mathbf{n}$ & $\%$ \\
\hline \multicolumn{3}{|l|}{ Sex } \\
\hline Female & 241 & 71 \\
\hline Male & 98 & 29 \\
\hline \multicolumn{3}{|l|}{ Age group } \\
\hline$\leqslant 30$ years & 54 & 16 \\
\hline $31-40$ years & 90 & 27 \\
\hline $41-50$ years & 98 & 29 \\
\hline$>50$ years & 97 & 29 \\
\hline \multicolumn{3}{|l|}{ Type of facility } \\
\hline Primary & 173 & 51 \\
\hline Secondary & 166 & 49 \\
\hline \multicolumn{3}{|l|}{ Employment in healthcare } \\
\hline$<10$ years & 178 & 53 \\
\hline $10-19$ years & 60 & 18 \\
\hline$\geqslant 20$ years & 101 & 30 \\
\hline \multicolumn{3}{|l|}{ Employment sector" } \\
\hline Provincial health dept & 209 & 63 \\
\hline City of Cape Town health dept & 23 & 7 \\
\hline Non-governmental organisation & 63 & 19 \\
\hline Other & 39 & 12 \\
\hline Health professional qualification & 131 & 39 \\
\hline Diabetes & 29 & 9 \\
\hline Current alcohol consumption & 109 & 32 \\
\hline \multicolumn{3}{|l|}{ Smoking history" } \\
\hline Nonsmoker & 216 & 64 \\
\hline Ex-smoker & 46 & 14 \\
\hline Current smoker & 76 & 22 \\
\hline Exposure to environmental tobacco smoke & 243 & 72 \\
\hline
\end{tabular}

Total $n=339$. All variables as measured at baseline 12 months earlier than the commencement of the follow-up phase. ${ }^{\#}: n=334 ;{ }^{\text {ๆ }}: n=338$.

\section{Test agreement at baseline}

There was only fair agreement between TST and QFT-GIT (concordance $72.1 \%$; $\kappa=0.29,95 \%$ CI 0.19 0.39 ) and between TST and T-SPOT.TB (concordance 68.1\%; $\kappa=0.27,95 \%$ CI $0.17-0.37$ ). Agreement analysis was performed between the IGRA assays and three different cut-offs for TST $(\geqslant 5, \geqslant 10$ and $\geqslant 15 \mathrm{~mm}$ ) without taking HIV status into consideration and was essentially unchanged across the different cut-off points (table 5). There was substantial agreement between the two IGRAs (concordance $84 \%$; $\kappa=0.65,95 \%$ CI $0.56-0.74)$.

\section{TST and IGRA conversions and annual incidence of TB infection}

The conversion rate represents the annual incidence of new TB infection as it measures new infections in those at risk after 1 year of observation. At follow-up testing, 13 out of 34 participants converted from a negative to a positive TST, representing an annual rate of infection of $38 \%$ (95\% CI $22-56 \%$ ), while $4 \%$ reverted from a previously positive to a negative test result.

For QFT-GIT, 25 out of 113 converted to a positive test, representing an annual rate of infection of $22 \%$ (95\% CI 15-31\%). The reversion rate was 7\%. For T-SPOT.TB, owing to a higher yield of indeterminate results, only 292 participants test results were utilised to generate conversion and reversion estimates. Of those who tested negative at baseline, 25 out of 114 converted their test response to positive representing a conversion rate of $22 \%$ (95\% CI $15-31 \%$ ). The reversion rate for this IGRA assay at $16 \%$ was considerably higher than that demonstrated by TST (table 6).

Subset analysis indicated no appreciable change in conversion or reversion rates for QFT-GIT (table 7). For T-SPOT.TB, however, there was a decline in conversion from $22 \%$ to $15 \%(p=0.31)$ and in the reversion rate from $16 \%$ to $14 \%(\mathrm{p}=0.68)$ in the sample when compared to the rates demonstrated for the larger group. These differences were, however, not statistically significant. 
TABLE 3 Prevalence of occupational and environmental risk factors among healthcare workers

\begin{tabular}{lcc} 
Variable & Subjects $\mathbf{~}$ & $\%$ \\
\hline Occupational tasks & & 49 \\
Interviewing TB patients & 166 & 43 \\
Counselling TB patients & 145 & 22 \\
Examination of TB patients & 74 & 22 \\
Collecting of sputum & 73 & 30 \\
Nursing TB patients & 103 & 9 \\
Homecare of TB patients & 29 & 2 \\
Bronchoscopy & 6 & 64 \\
Facility factors (self-reported) & & 93 \\
Infection control policy & 220 & 46 \\
Ventilation measures & 315 & 78 \\
Ultraviolet lights & 157 & 60 \\
Cough etiquette & 265 & 46 \\
Early triage & 205 & 77 \\
Separation of TB patients & 157 & 95 \\
Diagnostic services & 262 & 94 \\
Disposable surgical masks & 322 & 89 \\
N-95 masks & 317 & 65 \\
Personal protective equipment & 303 & 59 \\
Training (protection of self) & 219 & \\
Training (protection of patients) & 201 & \\
\hline
\end{tabular}

Total $n=339$. All variables as measured at baseline 12 months earlier than the commencement of the follow-up phase. TB: tuberculosis.

Different cut-off points were explored for IGRA conversion on account of the natural variability inherent in repeat use of these tests. For QFT-GIT, using the most stringent definition with a cut-off point of $0.70 \mathrm{IU} \cdot \mathrm{mL}^{-1}$, the conversion rate declined to $13 \%$, while for T-SPOT.TB, using an eight-spot increment, it declined to $18 \%$ (table 1 ).

\begin{tabular}{|c|c|c|}
\hline Variable & Subjects $\mathrm{n}$ & $\%$ \\
\hline Childhood BCG vaccination ${ }^{\#}$ & 313 & 92 \\
\hline Daily contact with TB patients ${ }^{\#}$ & 308 & 91 \\
\hline \multicolumn{3}{|l|}{ HIV status ( $n=329$ ) } \\
\hline Positive & 33 & 10 \\
\hline Negative & 296 & 90 \\
\hline TB symptom screen positive at follow-up & 55 & 16 \\
\hline \multicolumn{3}{|l|}{ CXR at follow-up ( $n=288)$} \\
\hline Normal & 242 & 84 \\
\hline Inactive TB & 41 & 14 \\
\hline Active TB & 5 & 2 \\
\hline Interval referral for TB/HIV & 44 & 13 \\
\hline History of TB treatment (ever TB) ${ }^{\#}$ & 44 & 13 \\
\hline Interim TB diagnosis & 7 & 2 \\
\hline Current TB diagnosis & 2 & 1 \\
\hline TST positive ${ }^{\#}(\mathrm{n}=329)$ & 275 & 84 \\
\hline TST positive ( $n=147$ ) & 123 & 84 \\
\hline QFT-GIT test positive ( $n=335$ ) & 231 & 69 \\
\hline T-SPOT.TB positive ( $\mathrm{n}=317)$ & 198 & 62 \\
\hline
\end{tabular}

CXR: chest radiograph. TST: tuberculin skin test; QFT-GIT: QuantiFERON-TB Gold-In-Tube. \#: variables as measured at baseline 12 months earlier than the commencement of the follow-up phase. 


\begin{tabular}{|c|c|c|c|}
\hline & $\mathrm{TST} \geqslant 5 \mathrm{~mm}$ & $\mathrm{TST} \geqslant 10 \mathrm{~mm}$ & $\mathrm{TST} \geqslant 15 \mathrm{~mm}$ \\
\hline \multicolumn{4}{|l|}{ QFT-GIT (n=482) } \\
\hline Positive TST and positive IGRA & $307(66)$ & $293(61)$ & $257(53)$ \\
\hline Negative TST and negative IGRA & $39(7)$ & $53(19)$ & $77(16)$ \\
\hline Positive TST and Negative IGRA & $126(26)$ & $112(23)$ & $88(18)$ \\
\hline Negative TST and positive IGRA & $10(2)$ & $24(5)$ & $60(12)$ \\
\hline Agreement \% & 71.8 & 71.8 & 69.3 \\
\hline Kappa $(95 \%$ CI) & $0.25(0.18-0.31)$ & $0.28(0.20-0.36)$ & $0.29(0.20-0.37)$ \\
\hline \multicolumn{4}{|l|}{ T-SPOT.TB (n=450) } \\
\hline Positive TST and positive IGRA & 259 (58) & 249 (55) & 221 (49) \\
\hline Negative TST and negative IGRA & $40(9)$ & 55 (12) & $84(19)$ \\
\hline Positive TST and Negative IGRA & $141(31)$ & $126(28)$ & $97(22)$ \\
\hline Negative TST and positive IGRA & $10(2)$ & $20(4)$ & $48(11)$ \\
\hline Agreement \% & 66.4 & 67.8 & 67.8 \\
\hline Kappa $(95 \%$ CI) & $0.21(0.14-0.28)$ & $0.25(0.16-0.33)$ & $0.30(0.21-0.39)$ \\
\hline
\end{tabular}

Data are presented as n (\%), unless otherwise stated. QFT-GIT: QuantiFERON-TB Gold-In-Tube.

Univariate and multivariate analysis of determinants of TST and IGRA conversion

Adjusted logistic regression models were applied to evaluate the relationship between each variable of interest and the conversion outcomes, treating age and sex as covariates (online data supplement).

In univariate analysis positive associations $(\mathrm{OR} \geqslant 2)$ were shown for TST conversion in those participants with the following characteristics: age $>40$ years, exposure to environmental tobacco smoke, engaging in collection of sputum, TB symptom screen positive at follow-up and those who had a referral for HIV care and or $\mathrm{TB}$ investigations in the screening interval, but none of these associations were statistically significant in multivariate analysis.

Following multivariate analysis, the following associations remained significant. Individuals engaged in counselling TB patients were less likely to have a TST conversion (OR 0.12, 95\% CI 0.15-0.92) while this same exposure variable emerged as a significant predictor of QFT-GIT conversion (OR 3.04, 95\% CI 1.01-9.15).

Positive predictors of T-SPOT.TB conversion were healthcare sector employment, individuals in local authority employment were 14 times more likely to convert than those working for the provincial health department (OR 14.19, 95\% CI 1.28-157.75), and having a positive TST at baseline (OR 3.40, 95\% CI $1.02-11.34)$.

\section{Discussion}

This is the first prospective study to evaluate test-specific annual infection rates in healthcare workers in a TB endemic country. The key findings were that: 1) there was a high prevalence of presumed LTBI in

TABLE 6 Results of latent tuberculosis infection (LTBI) testing at baseline and follow-up including annualised rates of conversion

\begin{tabular}{|c|c|c|c|}
\hline LTBI tests & TST & QFT-GIT & T-SPOT.TB \\
\hline \multicolumn{4}{|l|}{ Test status at baseline } \\
\hline Number with follow-up test result & 142 & 332 & 292 \\
\hline Number tested positive & $108(76)$ & $219(66)$ & $180(62)$ \\
\hline Number tested negative & $34(24)$ & 113 (34) & 115 (39) \\
\hline \multicolumn{4}{|l|}{ Conversion and reversion } \\
\hline Conversion rate & $13 / 34(38)$ & $25 / 113(22)$ & $25 / 115(22)$ \\
\hline Reversion rate & 4/108 (4) & $15 / 219(7)$ & $28 / 180(16)$ \\
\hline Annual rate of conversion ${ }^{\#} \%(95 \% \mathrm{CI})$ & $38(22-56)$ & $22(15-31)$ & $22(15-31)$ \\
\hline Annual rate of reversion $\%(95 \% \mathrm{CI})$ & $4(1-9)$ & $7(4-11)$ & $16(11-22)$ \\
\hline
\end{tabular}

Data are presented as $\mathrm{n}(\%)$, unless otherwise stated. TST: tuberculin skin test; QFT-GIT: QuantiFERON-TB Gold-In-Tube. \#: annual rate based on conversion rates in uninfected sample with repeat testing done 1 year later. 
TABLE 7 Subset analysis of conversion rates in group of healthcare workers who had tuberculin skin test (TST), QuantiFERON-TB Gold-In-Tube (QFT-GIT) and T-SPOT.TB tests performed concurrently at follow-up

\begin{tabular}{lcr} 
LTBI test & Conversion & \multicolumn{1}{c}{ Reversion } \\
\hline TST & $12 / 31(39 \%)(95 \% \mathrm{Cl} 22-58)$ & $4 / 93(4 \%)(95 \% \mathrm{Cl} 1-11)$ \\
QFT-GIT & $11 / 49(22 \%)(95 \% \mathrm{Cl} 12-37)$ & $5 / 75(7 \%)(95 \% \mathrm{Cl} 2-15)$ \\
T-SPOT.TB & $7 / 47(15 \%)(95 \% \mathrm{Cl} \mathrm{6-28)}$ & $11 / 77(14 \%)(95 \% \mathrm{Cl} 7-24)$
\end{tabular}

LTBI: latent tuberculosis infection.

healthcare workers irrespective of the test used $(>60 \%) ; 2)$ annual rates of new infection (conversion rates) were exceptionally high irrespective of the testing method used $(>20 \%)$; 3$)$ the conversion rate was substantially higher than those derived from community-based survey data, suggesting that TB infection was most likely occupationally acquired; 4) the rate of infection was significantly higher than that reported in healthcare workers in other TB-endemic countries, e.g. India; 5) IGRAs were characterised by significant reversion rates over 1 year (7-16\%); and 6) several occupational factors were identified that were significantly associated with test positivity.

The prevalence of LTBI based on TST was very high at $84 \%$ and similar to that of population-based studies of LTBI in South Africa using a TST value of $\geqslant 10 \mathrm{~mm}$ as cut-off $[19,20]$. An LTBI prevalence of $88 \%$ has been demonstrated in adults (non-healthcare workers) 31-35 years of age in Cape Town [20]. In contrast, lower rates of TST positivity have been reported in healthcare workers in high and intermediate $\mathrm{TB}$ incidence countries such as India, Vietnam and Georgia (40-67\%), using a TST cut-point of $\geqslant 10 \mathrm{~mm}$ [21-23]. Similarly, LTBI prevalence measured by QFT-GIT positivity (65\%) was similar to that in the community (56\%), and among healthcare workers in other high incidence countries (40-60\%) [19, 21-24]. The high rates of LTBI and apparent lack of differential prevalence between community and healthcare workers in our settings most likely reflects the extremely high background incidence of TB, high rates of exposure in the work environment, a high prevalence of HIV co-infection increasing susceptibility, and the inclusion of non-governmental organisation staff which were, in the main, drawn from high TB prevalence communities.

The annual incidence of infection in this population was found to be extraordinarily high at between 20 and $38 \%$ depending on the assay used. While there are no comparable studies among South African adults, a recent study among adolescents in a high HIV prevalence community in Cape Town reported a high annual incidence of infection of $11 \%$ [25]. The authors argue that social contact is likely to be at its peak during adolescence and that risk of TB infection is more likely to resemble that of adults than children in the community. Using this estimate as an adult proxy measure, healthcare workers in our study were 3.5 times more likely to become infected during a 1-year period than a community-based comparison group. This is likely to reflect increased risk due to occupational exposure to TB. This annual risk was much higher (5-9 times) than demonstrated in two Indian healthcare worker-specific studies in a high TB incidence setting $[1,26]$.

IGRA-related conversion rates were lower than those using TST, but variable. The optimal cut-off point for conversion when using IGRAs for serial screening remains unclear, irrespective of the setting [27, 28]. The role of natural within subject variability, high reversion rates $(7-16 \%$ with the IGRAs versus only $4 \%$ with TST), and a possible boosting effect on IGRAs by TST may explain part of this variability [29]. Recent published data using serial IGRAs in healthcare workers from the USA showed high rates of reversion in those that had apparently converted, thus calling into question how serial testing is currently performed and what cut-off points are used [30, 31]. More data are also needed to accurately define the "grey zone" around the cut-off point currently in use for QFT-GIT and T-SPOT.TB assays [17].

An increased risk of TST conversion in healthcare settings has been associated with occupational category, occupational setting and increasing age [32]. However, in our study there were no factors consistently associated with test conversion of either the TST or the IGRAs. The three-fold increased risk for QFT-GIT conversion associated with counselling of TB patients was the reverse of the negative association with TST conversion. This suggests that the test outcomes are not equivalent and calls into question the suggested substitution of TST with IGRAs in this population. The strong association between a baseline positive TST result and a later T-SPOT.TB conversion points to possible suboptimal sensitivity of the T-SPOT.TB. This association may also reflect marked variability of this assay, emphasising the need to re-examine cut-off points denoting conversion [17]. The strong association between T-SPOT.TB conversion (OR 14.19) and 
employment by the local authority is suggestive of a causative role for occupational exposure, as the provision of TB services is a function of local authority clinics and staff. The lack of a protective effect of occupational and environmental control measures on conversion rates may be ascribed to a reliance on reported rather than actual implementation of such measures or the failure to ensure the effectiveness of such interventions (e.g. failure to implement triage plans or maintenance of ultraviolet lights and ventilations systems). The lack of association between childhood BCG vaccination and TST positivity is consistent with the demonstrated lack of BCG effect on TST response when administered at birth [33].

There were several limitations to our study. This study was subject to volunteer selection bias at both the baseline and follow-up phase. Nurses and doctors at greater risk of TB infection were under-represented as a result of their limited time to participate in the study. The inclusion of HIV testing as part of the protocol may have discouraged participation due to stigma. Loss to follow up was a significant problem and probably led to attrition bias, the impact of which is unclear. In keeping with the healthy worker participation effect, retested participants were older, with longer length of service, and more likely to be HIV negative. Loss to follow up was also related to the mobility and high staff turnover in the non-governmental organisation sector. Since the study setting is one of the highest TB incidence areas in the country, the findings may not be generalisable to all healthcare workers in South Africa or to other high incidence occupational settings. The reliance on self-reported measures of occupational exposure also made it difficult to arrive at accurate measures of such exposure and its relationship with presumed LTBI.

In summary, this study demonstrates a very high prevalence of LTBI using different test modalities in healthcare workers in our setting. This very high annual incidence of infection is strongly suggestive of a causative role for occupational exposure. Nevertheless, the role and mechanisms of occupational exposure in driving TB infection among healthcare workers in TB endemic countries requires further exploration as effective infection control measures would have to be informed by a clear understanding of such factors.

\section{Acknowledgements}

Technical support: Vivienne Woodburne for laboratory support and processing of T-cell assays. Data collection: Elize Pietersen and Lameze Abrahams for conducting the fieldwork for the data collection (Lung Infection and Immunity Unit, Dept of Medicine, University of Cape Town, Cape Town, South Africa).

\section{References}

1 Christopher DJ, James P, Daley P, et al. High annual risk of tuberculosis infection among nursing students in South India: a cohort study. PLoS One 2011; 6: e26199.

2 Baussano I, Nunn P, Williams B, et al. Tuberculosis among health care workers. Emerg Infect Dis 2011; 17: 488-494.

3 Joshi R, Reingold AL, Menzies D, et al. Tuberculosis among health-care workers in low- and middle-income countries: a systematic review. PLoS Med 2006; 3: e494.

4 Menzies D, Joshi R, Pai M. Risk of tuberculosis infection and disease associated with work in health care settings. Int J Tuberc Lung Dis 2007; 11: 593-605.

5 Balt E, Durrheim D, Weyer K. Nosocomial transmission of tuberculosis to health care workers in Mpumalanga. S Afr Med J 1998; 88: 1363-1364.

6 Wilkinson D, Gilks C. Increasing frequency of tuberculosis among staff in a South African District hopsital:impact of the HIV epidemic on the supply side of health care. Trans R Soc Trop Med Hyg 1998; 92: 500-502.

7 Naidoo S, Jinabhai C. TB in health care workers in Kwazulu-Natal, South Africa. Int J Tuberc Lung Dis 2006; 10: 676-682.

8 O'Donnell MR, Jarand J, Loveday M, et al. High incidence of hospital admissions with multidrug-resistant and extensively drug-resistant tuberculosis among South African health care workers. Ann Intern Med 2010; 153: 516-522.

9 Jarand J, Shean K, O'Donnell M, et al. Extensively drug-resistant tuberculosis (XDR-TB) among health care workers in South Africa. Trop Med Int Health 2010; 15: 1179-1184.

10 South Africa. Dept. of Health (1994-). Human Resources for Health South Africa: HRH Strategy for the Health Sector, 201213\2013 201617. Pretoria, Dept of Health, 2011.

11 World Health Organization. International Labour Organization. Joint WHO/ILO policy guidelines on improving health worker access to prevention, treatment and care services for HIV and TB. Geneva, World Health Organization, 2010.

12 Zungu M, Malotle M. Do we know enough to prevent occupationally acquired tuberculosis in health care workers. Occup Health S Afr 2011: 17-21.

13 Pai M. Alternatives to the tuberculin skin test: interferon-gamma assays in the diagnosis of mycobacterium tuberculosis infection. Indian J Med Microbiol 2005; 23: 151-158.

14 Mazurek GH, Jereb J, Vernon A, et al. Updated guidelines for using interferon gamma release assays to detect Mycobacterium tuberculosis infection - United States, 2010. MMWR Recomm Rep 2010; 59: 1-25.

15 National Collaborating Centre for Chronic Conditions (UK). Centre for Clinical Practice at NICE (UK). Tuberculosis: Clinical Diagnosis and Management of Tuberculosis, and Measures for Its Prevention and Control. London, National Institute for Health and Clinical Excellence (UK), 2011.

16 Pinto LM, Dheda K, Theron G, et al. Development of a simple reliable radiographic scoring system to aid the diagnosis of pulmonary tuberculosis. PLoS One 2013; 8: e54235.

17 van Zyl-Smit RN, Zwerling A, Dheda $\mathrm{K}$, et al. Within-subject variability of interferon-g assay results for tuberculosis and boosting effect of tuberculin skin testing: a systematic review. PLoS One 2009; 4: e8517. 
18 American Thoracic Society. Targeted tuberculin testing and treatment of latent tuberculosis infection. $M M W R$ Recomm Rep 2000; 49: 1-51.

19 Mahomed H, Hughes EJ, Hawkridge T, et al. Comparison of mantoux skin test with three generations of a whole blood IFN-gamma assay for tuberculosis infection. Int J Tuberc Lung Dis 2006; 10: 310-316.

20 Wood $\mathrm{R}$, Liang $\mathrm{H}, \mathrm{Wu} \mathrm{H}$, et al. Changing prevalence of tuberculosis infection with increasing age in high-burden township in South Africa. Int J Tuberc Lung Dis 2010; 14: 406-412.

21 Pai M, Gokhale K, Joshi R, et al. Mycobacterium tuberculosis infection in health care workers in rural India: comparison of a whole-blood interferon gamma assay with tuberculin skin testing. JAMA 2005; 293: 2746-2755.

22 Mirtskhulava V, Kempker R, Shields KL, et al. Prevalence and risk factors for latent tuberculosis infection among health care workers in Georgia. Int J Tuberc Lung Dis 2008; 12: 513-519.

23 Lien LT, Hang NT, Kobayashi N, et al. Prevalence and risk factors for tuberculosis infection among hospital workers in Hanoi, Viet Nam. PLoS One 2009; 4: e6798.

24 Drobniewski F, Balabanova Y, Zakamova E, et al. Rates of latent tuberculosis in health care staff in Russia. PLoS Med 2007; 4: e55.

25 Middelkoop K, Bekker LG, Myer L, et al. Rates of tuberculosis transmission to children and adolescents in a community with a high prevalence of HIV infection among adults. Clin Infect Dis 2008; 47: 349-355.

26 Pai M, Joshi R, Dogra S, et al. Serial testing of health care workers for tuberculosis using interferon- $\gamma$ assay. Am J Resp Crit Care Med 2006; 174: 349-355.

27 Pai M, O'Brien R. Serial testing for tuberculosis: can we make sense of $\mathrm{T}$ cell assay conversions and reversions? PLoS Med 2007; 4: e208.

28 Zwerling A, Benedetti A, Cojocariu M, et al. Repeat IGRA testing in Canadian health workers: conversions or unexplained variability? PLoS One 2013; 8: e54748.

29 van Zyl-Smit RN, Pai M, Peprah K, et al. Within-subject variability and boosting of T-cell interferon-gamma responses after tuberculin skin testing. Am J Respir Crit Care Med 2009; 180: 49-58.

30 Fong KS, Tomford JW, Teixeira L, et al. Challenges of interferon-gamma release assay conversions in serial testing of health-care workers in a TB control program. Chest 2012; 142: 55-62.

31 Dorman SE, Belknap R, Graviss EA, et al. Interferon-gamma release assays and tuberculin skin testing for diagnosis of latent tuberculosis infection in healthcare workers in the United States. Am J Respir Crit Care Med 2014; 189: 77-87.

32 Baussano I, Bugiani M, Carosso A, et al. Risk of tuberculin conversion among healthcare workers and the adoption of preventive measures. Occup Environ Med 2007; 64: 161-166.

33 Farhat M, Greenaway C, Pai M, et al. False-positive tuberculin skin tests: what is the absolute effect of BCG and non-tuberculous mycobacteria? Int J Tuberc Lung Dis 2006; 10: 1192-1204. 